\title{
ANTECEDENTES DE LOS TALLERES DE ARQUITECTURA: UNA COLABORACIÓN ENTRE DOCENTES
}

\author{
Casares Gallego, Amparo'; Raya De Blas, Antonio² \\ 'Departamento de Proyecto, Urbanismo y Composición Arquitectónica, \\ Escuela Técnica Superior de Arquitectura. \\ ${ }^{2}$ Departamento de Construcciones y Estructuras arquitectónicas, de edificación y \\ aeronáuticas, Escuela Técnica Superior de Arquitectura
}

\section{RESUMEN}

Se recogen los antecedentes de los Talleres de Arquitectura, experiencia de colaboración entre profesores, que gestionan la transversalidad de la naturaleza del oficio de la arquitectura. Éstos fueron concebidos para facilitar la confluencia de los contenidos de las diferentes asignaturas en torno al proyecto arquitectónico, garantizando la optimización de los recursos docentes y racionalizando el trabajo del alumnado. El modelo propuesto es un espacio de trabajo e intercambio al que asisten simultáneamente, no secuencialmente, los profesores de las diversas materias: proyectos, construcción, estructuras, urbanística, instalaciones y composición. Estos talleres están ahora incorporados en los nuevos planes de estudios de la titulación: grado y máster. Aquí se exponen y analizan los antecedentes, las experiencias previas, que determinaron la asunción de este modelo.

\section{PALABRAS CLAVE}

Taller de Arquitectura, docencia transversal, colaboración entre docentes. 


\section{CITA RECOMENDADA:}

Casares Gallego, A., Raya De Blas, A.(2018). Antecedentes de los talleres de arquitectura: una colaboración entre docentes. En E. de la Torre Fernández (ed.) (2018). Contextos universitarios transformadores: retos e ideas innovadoras. II Xornadas de Innovación Docente. Cufie. Universidade da Coruña (pág. 331-350).

DOI capítulo: https://doi.org/10.17979/spudc.9788497496780.331

DOI libro: https://doi.org/10.17979/spudc.9788497496780

\section{ABSTRACT}

The history of the Architecture Workshops (Talleres de Arquitectura) is revised, where these workshops are a collaboration experience among professors who manage the transversal nature of the architecture trade. These workshops were conceived to facilitate the confluence of the contents of the different subjects that concern the architectural project, thus guaranteeing the optimization of the teaching resources and rationalizing the work of the students. The proposed model is a work and exchange space that is attended simultaneously, not sequentially, by the professors of the different subjects: projects, construction, structures, urban planning, installations and composition. These workshops are now incorporated in the new curricula of the School of Architecture: degree and master. Here the antecedents, the previous experiences that determined the assumption of this model, are exposed and analysed.

\section{KEY WORDS}

Architecture workshop, transversal teaching, professors collaboration. 


\section{INTRODUCCIÓN}

La Escuela Técnica Superior de Arquitectura (ETSAC) imparte una titulación profesionalizante cuyo objetivo es la capacidad de idear, de imaginar un lugar deseable para el habitar humano en cualquiera de sus acepciones.

La enseñanza académicamente de la arquitectura' se ha estructurado secularmente como una suerte de disciplinas orientadas y alrededor del Proyecto Arquitectónico. Estas disciplinas como la expresión gráfica, la física, las matemáticas, la composición, la historia del arte, la construcción, el urbanismo, las estructuras, las instalaciones o la legislación han sido presididas por la creación arquitectónica, como elemento ordenador y aglutinante de todas ellas.

Los primeros estudios de Arquitectura se impartieron en la Academia de Matemáticas, creada por Felipe II en 1582, donde existió una efímera cátedra de Arquitectura. Tras diversos intentos la Real Academia de las tres nobles artes de San Fernando estableció los estudios de Arquitectura en 1752 y en 1844 la antigua sala de Arquitectura de San Fernando se conoció como "escuela especial" de Arquitectura. En el reglamento de 1845 se consolidó la desvinculación de la docencia de esta "escuela" de Arquitectura respecto a la de pintura y escultura.

En el plan generado por el decreto de 1858 para los estudios de arquitectura se establece una parte común sin carácter politécnico, sino de transversalidad interdisciplinar donde los tres primeros cursos se realizaban en la Facultad de Ciencias. Paralelamente los estudiantes debían adquirir la destreza gráfica que le permitiese superar el examen de ingreso en la Escuela Especial de Arquitectura y cursar cinco años de disciplinas específicas. Este esquema de transversalidad permaneció en vigor casi un siglo. Y es en 1957 es cuando se implanta el Proyecto Fin de Carrera (PFC) en el plan de estudios, materia que será tratada en esta exposición. Y es en 1964 es cuando el plan elimina la obligación de cursar estudios en la Facultad de Ciencias y en otras Escuelas Técnicas, terminando con la transversalidad. 
Las características de las disciplinas y la orientación profesional del título aparejaban la impartición por un profesorado con una dilatada y cualificada experiencia profesional, fundamentalmente en la materia de proyectos arquitectónicos y disciplinas específicas. Criterio que ha cambiado por la deriva de la Universidad hacia un modelo académico puro frente a un académico con contrastada experiencia profesional, algo fundamental en las enseñanzas profesionalizantes.

En estas décadas se ha procedido a una lenta pérdida de la transversalidad en la formación y de la profesionalidad de sus docentes, cada vez más académicos, con un incremento en la complejidad técnica de la profesión que ha jugado contra la creatividad.

El proyecto y la creación arquitectónica han estado presentes permanentemente como bandera de la docencia en arquitectura, pero la fragmentación del proceso de aprendizaje y la especialización de las disciplinas han difuminado la transmisión del objetivo de la profesión de arquitecto: el proyecto. La detección de esta casuística empujo a las diferentes asignaturas a solicitar a sus alumnos un proyecto arquitectónico específico independiente para aplicar su docencia, sin coordinación entre ellas, ni con la asignatura de proyectos arquitectónicos. Es cierto que la consecución del hecho arquitectónico se realizaba desde disciplinas diferentes, pero sin la transversalidad y unicidad necesarias. Todo esto provoco una sobrecarga del esfuerzo de los estudiantes dentro de una estructura docente anual.

En este contexto las cifras de fracaso escolar eran preocupantes, tal como se aprecia en la tabla1"ii adjunta extraída del plan de Grado en Estudios en Arquitectura de 2014. 


\begin{tabular}{|c|c|c|c|c|c|c|c|c|c|c|}
\hline \multicolumn{11}{|c|}{ INDICADORES DE TITULACIONES DE LA ETSAC (duración 5 años + PFC) } \\
\hline & CURSO & $\begin{array}{l}2005 / 0 \\
6\end{array}$ & $\begin{array}{l}2006 / 0 \\
7\end{array}$ & $\begin{array}{l}2007 / 0 \\
8\end{array}$ & $\begin{array}{l}2008 / 0 \\
9\end{array}$ & $\begin{array}{l}2009 / 1 \\
0\end{array}$ & $\begin{array}{l}2010 / 1 \\
1\end{array}$ & $\begin{array}{l}2011 / 1 \\
2\end{array}$ & $\begin{array}{l}2012 / 1 \\
3\end{array}$ & $\begin{array}{l}2013 / 1 \\
4\end{array}$ \\
\hline \multirow{2}{*}{$\begin{array}{l}\text { Estudiantes } \\
\text { matriculado } \\
\text { s }\end{array}$} & $\begin{array}{l}\text { Arquitect } \\
0\end{array}$ & 2109 & 2083 & 2016 & 1936 & 1898 & 1648 & 1411 & 1175 & 946 \\
\hline & Grado & & & & & & 245 & 477 & 671 & 814 \\
\hline \multirow{2}{*}{$\begin{array}{l}\text { Tasa de } \\
\text { abandono }\end{array}$} & $\begin{array}{l}\text { Arquitect } \\
0\end{array}$ & $22,08 \%$ & $18,97 \%$ & $29,28 \%$ & $23,85 \%$ & $19,83 \%$ & $25,81 \%$ & $13,59 \%$ & $18,36 \%$ & $1,13 \%$ \\
\hline & Grado & & & & & & & & $7,5 \%$ & $8,9 \%$ \\
\hline \multirow{2}{*}{$\begin{array}{l}\text { Tasa de } \\
\text { eficiencia }\end{array}$} & $\begin{array}{l}\text { Arquitect } \\
0\end{array}$ & $66,32 \%$ & $67,23 \%$ & $68,16 \%$ & $70,10 \%$ & $69,36 \%$ & $66,73 \%$ & $69,30 \%$ & $70,14 \%$ & $70,37 \%$ \\
\hline & Grado & & & & & & $100 \%$ & $92,49 \%$ & $86,71 \%$ & $83,43 \%$ \\
\hline $\begin{array}{l}\text { Tasa de } \\
\text { graduación }\end{array}$ & $\begin{array}{l}\text { Arquitect } \\
0\end{array}$ & $9,68 \%$ & $11,27 \%$ & $6,76 \%$ & $6,22 \%$ & $10,96 \%$ & $11,82 \%$ & $11,34 \%$ & $6,00 \%$ & $0,52 \%$ \\
\hline \multirow{2}{*}{$\begin{array}{l}\text { Tasa de } \\
\text { rendimiento }\end{array}$} & $\begin{array}{l}\text { Arquitect } \\
0\end{array}$ & $54,52 \%$ & $55,24 \%$ & $58,77 \%$ & $58,69 \%$ & $61,13 \%$ & $62,60 \%$ & $63,23 \%$ & $60,11 \%$ & $42,09 \%$ \\
\hline & Grado & & & & & & $74,20 \%$ & $68,38 \%$ & $67,47 \%$ & $61,06 \%$ \\
\hline $\begin{array}{l}\text { Duración } \\
\text { Media } \\
\text { (años) }\end{array}$ & $\begin{array}{l}\text { Arquitect } \\
0\end{array}$ & 7,73 & 8,38 & 8,47 & 8,66 & 8,37 & 8,22 & 8,17 & 8,27 & 8,49 \\
\hline
\end{tabular}

Tabla 1 Indicadores de las titulaciones impartidas en 2014 en la ETSAC.

Memoria verificada de Grado en Estudios en Arquitectura

Por otra parte, estas deficiencias afloraban con claridad en el Proyecto Fin de Carrera del título de Arquitecto, asignatura de solo 3 créditos, sin enseñanza reglada, pero con una duración de más un año de tutorías constantes con un arquitecto docente. Esta "asignatura" de PFC se propone como un "proyecto integral de arquitectura de naturaleza profesional en el que se sinteticen todas las competencias adquiridas en la carrera, desarrollado hasta el punto de demostrar suficiencia para determinar la completa ejecución de las obras de edificación sobre las que verse, con cumplimiento de la reglamentación técnica y administrativa aplicable." (Orden EDU 2075/2010)

La Arquitectura que deseamos ha de hacer confluir en el Proyecto Arquitectónico, la solución precisa y única de los conocimientos adquiridos en las diversas asignaturas específicas - 
proyectos, urbanismo, estructuras, construcción e instalaciones- y todo dentro de un grafismo cuidado, actualizado a los sistemas de información del momento.

Las características de los estudios y la orientación profesional del Título de Arquitecto conllevan una formación amplia en régimen de prácticas y/o laboratorios. A partir del tercer curso de estudios la enseñanza a través de la práctica se intensifica, en este momento ya se han adquirido las herramientas del oficio (disciplinas propedéuticas como expresión gráfica, física, matemáticas).

Como solución a esta compleja situación surge una respuesta del profesorado en el curso 2008-2009, en cuarto curso de la titulación de Arquitecto. Comenzamos una experiencia de colaboración de cuatro asignaturas (Proyectos 4, Construcción 4, Estructuras 3 y Urbanística 3) con diverso resultado en las asignaturas. Se pactaba alrededor del programa de la asignatura de proyectos el área a trabajar en urbanismo, alguna peculiaridad estructural en hormigón que correspondía a esta asignatura y una envolvente constructiva que satisfacía el contenido de la construcción de este año de carrera.

Muchos inconvenientes hacían difícil obtener resultados positivos. Por ejemplo, la variable situación de matrícula del alumnado: pocas personas compartían en cuarto curso las cuatro asignaturas. Otro escollo era la incomodidad que representaba esta situación para parte del profesorado. Aún en este escenario, a partir del segundo año se empezó a notar que el modelo tenía futuro, se producían proyectos mejores y más completos, lo que deparaba más aprobados.

Cuando en el año 2010 empieza la extinción del título de Arquitecto, la matrícula en PFC es de más de 600 estudiantes, con un porcentaje elevado de "larga duración" (más de 10 años matriculándose), aunque muchos de ellos no realizaban ninguna labor al respecto. Ante esta situación se plantea un modelo interdisciplinar de docencia en PFC más ambicioso para este alumnado de" larga duración". En este modelo interdisciplinar confluían profesores de las distintas asignaturas específicas de la carrera coordinados por profesores de proyectos arquitectónicos. La asistencia del alumnado a las sesiones conjuntas semanales era 
obligatoria. Estableciéndose, también, clases teóricas de apoyo y reciclaje de estos estudiantes, abiertas a todos los estudiantes de PFC. Este modelo interdisciplinar de PFC duro cinco años.

En cuarto curso se experimentó con la colaboración de docentes alrededor de un tema de proyectos con puestas en común, pero con docencia totalmente diferenciada. Y en PFC con un modelo interdisciplinar de docencia simultánea.

Este modelo de colaboración en cuarto curso y de docencia en PFC, ambos con resultados exitoso, serán el germen de los TALLERES para la docencia en Grado y Proyecto Fin de Carrera del Grado en Arquitectura (titulación del 2010) y del Trabajo Fin Máster del Máster Universitario en Arquitectura (titulación de 2015). En ambas experiencias hemos participado como subdirectores de la ETSAC y docentes de las asignaturas durante años.

\section{ANTECEDENTES}

Aunque con el nombre de Taller de Arquitectura podemos encontrar diferentes experiencias docentes (alguna de marcado carácter político como el "Taller total"iii en Córdoba, Argentina), nos vamos a centrar en experiencias más cercanas y coincidentes con el planteamiento del Taller de Arquitectura (TA) de la ETSAC.

La Escuela Técnica Superior de Arquitectura del Valles (ETSAV) en su Plan de Estudios (1994), plantea la singularidad de la asignatura de Talleres de Arquitectura y Proyectos (TAP), espacios pedagógicos de encuentro entre la asignatura de proyectos y el resto de asignaturas del curso. Este planteamiento docente es el germen de las experiencias desarrolladas desde el año 2008 en cuarto curso de la ETSAC y en PFC, también, del plan de Grado en Arquitectura implantado en al año 2010 aunque con sustanciales diferencias.

En ambos casos, ETSAC y ETSAV se producía una obligada colaboración entre los docentes adscritos al taller, pero en la ETSAV se trata de una asignatura. Una vez alcanzada la necesaria situación de equilibrio, creemos que ha demostrado ser un vehículo de acercamiento entre los 
docentes que, de alguna manera, produce una intensidad de conocimiento que revierte en beneficio del estudiantado.

Los resultados de Taller para estudiantes de "larga duración" ha permitido disolver la bolsa de estudiantes que desde el plan del 1975 estaban pendiente de la presentación y superación del PFC, aunque haya hundido la tasa de duración media en años de la titulación.

La incorporación del "Concurso Multiconfort House" al trabajo coordinado de las asignaturas de cuarto curso del título de arquitecto en el año 2010 y posteriormente (2014) al Taller de Arquitectura del mismo curso, donde participan universidades de todo el estado, nos ha permitido cosechar un considerable éxito contrastado. iv

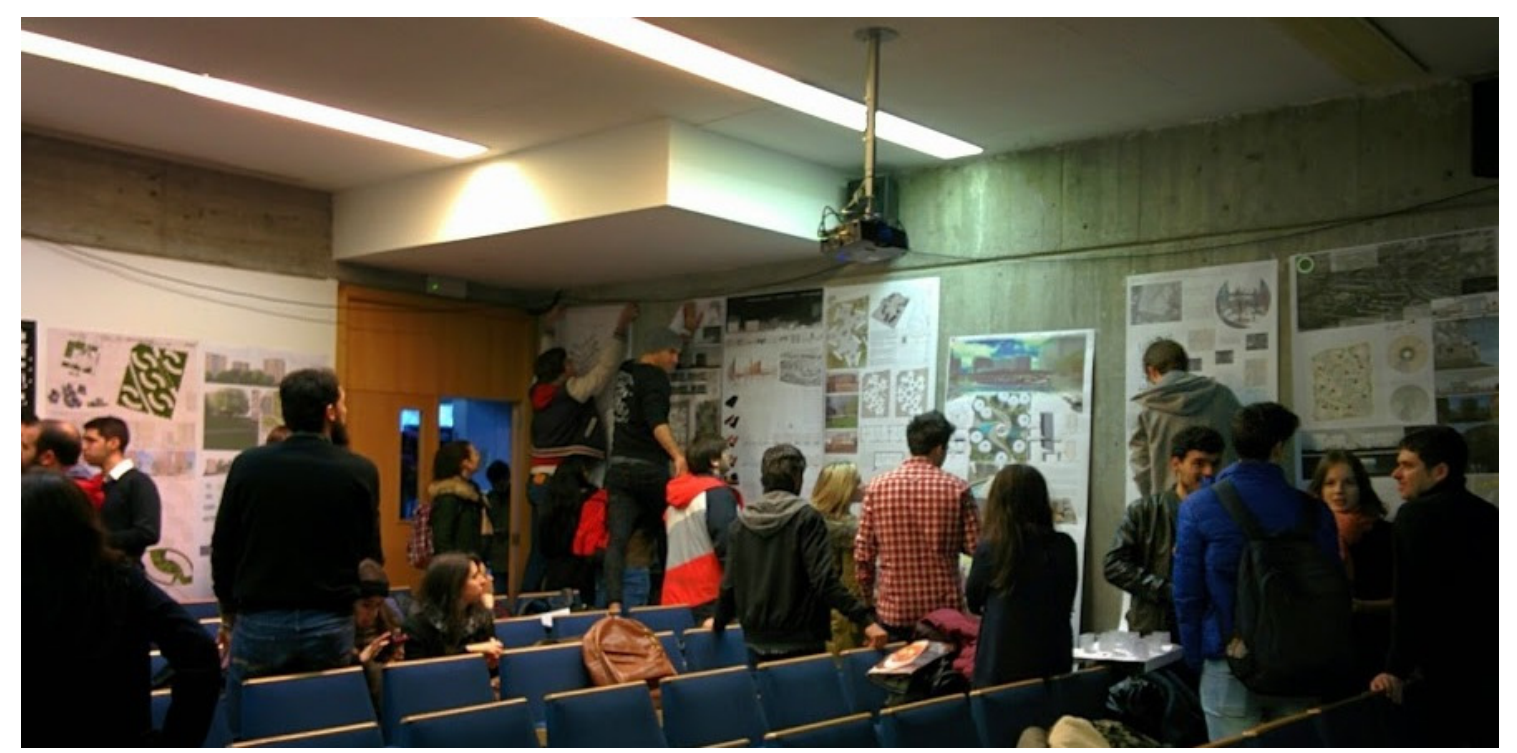

Imagen 1 Exposición de trabajos para el "Concurso Multiconfort House". Salón de Actos de la ETSAC Foto de la autora. 


\section{EL TALLER DE ARQUITECTURA EN LA ETSAC}

Así cuando, obligadamente, se formula el Plan de Grado en Arquitectura (2010), se aprovechan las citadas experiencias, entre otras similares, para proponer el modelo TA como elemento vertebrador de la nueva titulación. Estructura que se ha mantenido en el Grado de Estudios de Arquitectura y el Máster Universitario en Arquitectura que cumplimentan la orden EDU 2075/2010, que determina la estructura de los estudios conducentes a la profesión de Arquitecto actualmente. Este TA se implementó en todos los curso y semestres en la implantación de 2010 y no se planeó para el primer semestre en el caso del Plan del 2015.

\subsection{Descripción del Taller de Arquitectura}

EI TA de la Escuela Técnica Superior de Arquitectura de A Coruña queda definido en la memoria verificada del Grado en Arquitectura por la UDC de 2009 como "un espacio de trabajo e intercambio concebido para facilitar la confluencia de los contenidos de las diferentes asignaturas en torno al proyecto arquitectónico, garantizando la optimización de los recursos docentes y racionalizando el trabajo del alumno. El Taller pretende establecer mecanismos de coordinación y transversalidad a lo largo de los estudios, evitando duplicidades y reiteración en los contenidos, facilitando el tránsito eficaz del alumno entre los sucesivos semestres, paliando la incidencia negativa que la dispersión de asignaturas cursadas en diferentes cursos por gran parte del alumnado tiene en la exigible eficiencia del sistema docente."

Por tanto, no se trata de una asignatura, como la experiencia del TAP de la ETSAV. Se busca un nuevo modelo que solvente reticencias e imposibilidades técnicas. El aprendizaje realizado con las experiencias de cuarto curso y PFC aconsejaba mantener la independencia de las diferentes asignaturas, amén de solventar limitaciones de carácter burocrático inherentes al Sistema Universitario Gallego (número de ECTS de las asignaturas de Taller, condicionantes de matrícula o proceso de actas).

Se establece, en la memoria de la titulación, que cada asignatura debe de aportar una serie de créditos prácticos de su docencia a este Taller de Arquitectura. Así, se conforma un espacio 
de trabajo, con diferentes docentes simultáneos, de diferentes disciplinas, alrededor de un tema de la asignatura proyectos arquitectónicos pactado. Se repetía la descripción utilizada en la experiencia de cuarto curso.

En la versión del grado de 2010, la totalidad de la asignatura de Proyectos Arquitectónicos, se integraba en el TA, condicionando su autonomía y posibles líneas de docencia específicas de proyectos.

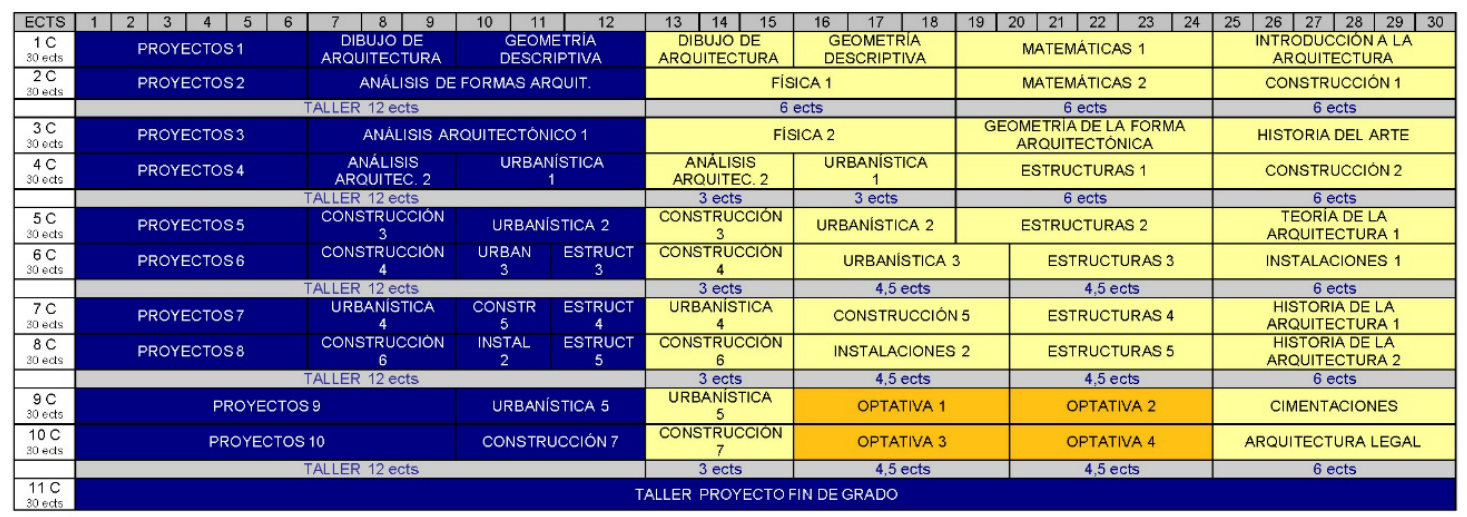

TOTAL 330 ECTS

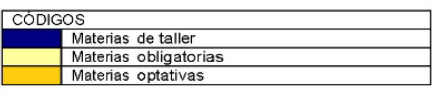

Tabla 2 Esquema del plan de estudios Grado en Arquitectura del 2010, en la ETSAC

Este criterio fue modificado en el plan de 2015, igualando la participación entre asignaturas incluso proyectos en el TA.

La problemática detectada en la experiencia de cuarto curso mostraba una matrícula desigual, muy poco homogénea que impedía la aplicación de esta experiencia educativa. La propia estructura de la docencia en arquitectura por disciplinas fomenta la aparición del tipo de estudiante entusiasta de los proyectos, de la construcción o de las estructuras que cursaba en vertical la docencia, olvidando el resto de las asignaturas hasta el final. No solo era un problema para esta nueva propuesta docente sino para la formación de calidad de los futuros arquitectos en un plazo razonable. 
Era necesario articular una norma que permitiese, desde el comienzo del plan, evitar esta verticalidad y garantizar unos estudios horizontales que permitiesen la docencia en TA.

Siendo imposible la incompatibilidad entre asignaturas y para garantizar la horizontalidad en el desarrollo del Plan de estudios, se establecieron las siguientes condiciones para cursar los Talleres de Arquitectura en los diferentes semestres:

"Los alumnos tendrán que cursar simultáneamente todas las asignaturas del Taller, por lo que sí es la primera vez que se matriculan en asignaturas de un taller tendrán que hacerlo en todas las asignaturas del mismo".

"Los alumnos tendrán que cursar previa o simultáneamente a un taller todas las asignaturas vinculadas a talleres anteriores que no hayan superado completamente".

Estas condiciones, contestadas por el estudiantado, conseguían la horizontalidad en la que todos los estamentos -docentes y discentes- estábamos de acuerdo. Condiciones que se mantuvieron en el plan del 2015 ante la imposibilidad de encontrar otra solución mejor.

Destacar que se exige cursar las asignaturas del Taller de Arquitectura, no solo matricularse. Este punto ha sido fuente de disfunciones entre el estudiantado y el propio profesorado.

Frente a estas facetas negativas pero necesarias, el cursar el TA con sus diferentes asignaturas permitía recuperar en diferentes oportunidades y semestres las deficiencias del "proyecto del Taller" que computaba como práctica de todas las asignaturas, evitando los bloqueos en el avance del estudiante, en aras de la misma horizontalidad invocada anteriormente.

Es necesario la confluencia de estudiantes cursando las mismas asignaturas para que esta formación transversal sea posible con la simultaneidad de docentes de diferentes disciplinas. Una vez ocurrida esta coincidencia la posible no superación de una aparte de una asignatura del TA no podía retrasar al estudiante en su formación y acceso al siguiente TA. Por otra parte, la presencia de estudiantes con matrícula parcial en el TA, procedente de la ausencia de 
ciertas competencias era tan distorsionador como la "matricula vertical". Este problema podía ser solventado en tutorías personalizadas.

El Taller de Arquitectura de PFC no plantea este problema al tratarse como una asignatura de TFG (2010) o TFM (2015) de 30 ECTS (según orden EDU2075/2010). Esta asignatura tiene asignada docencia a diferentes disciplinas específicas de la arquitectura. Todo ello está recogido en un Reglamento específico de PFC. vi

\subsection{Funcionamiento del TA}

La coordinación de los TA recae en los profesores de proyectos, con alguna excepción en los primeros semestres. Esta asignación se repite en el TA de PFC. Cada grupo de Taller está compuesto por un número variable de profesores, entre 3 y 4 en el grado y 7 en los Talleres de PFC. Los temas de cada TA son acordados entre los profesores de acuerdo con una temática especificada en el plan de Grado 0 en con unas líneas de desarrollo acordadas en el TFM. Los temas de los TA de PFC son publicados antes de la matrícula de los estudiantes.

La asignación de los estudiantes a los diferentes TA de PFC se realiza por elección del tema propuesto 0 por afinidad con el grupo de profesores que proponen el tema. Finalmente existe un criterio curricular para la asignación del alumnado a los TA de PFC y garantizar el equilibrio en la ratio profesor /alumno, siempre discutible.

El desarrollo de la docencia en estos TA es enriquecedor. El profesorado aporta diferentes visiones alrededor de la temática propuesta, con clases expositivas de apoyo y con respuesta a las diferentes propuestas del estudiantado.

Los trabajos individuales finales se complementan con estudios iniciales en grupo sobre el tema de trabajo del TA. Así, al existir homogeneidad entre los estudiantes es posible crear grupos de forma dinámica y promover el aprendizaje colaborativo, compartiendo información y apoyando resultados.

Las sesiones conjuntas de exposición pública de los enfoques iniciales del alumnado 0 de sus propuestas se suceden con debates participativos e interdisciplinares del PDI. La 
transversalidad y la unicidad de la finalidad están presentes a la vez que se elimina carga de trabajo para el estudiante en aras de una mayor calidad en su adquisición de habilidades y competencias.

Las visitas a obras singulares aportan un complemento de realidad en la formación de estos estudiantes, aunque estas se suelen concretar en el Taller de PFC. Estas visitas, independientemente de los comentarios de los docentes, aportan puntos de vista nuevo: del arquitecto que ha proyectado y construido la obra.

Las sesiones de exposición pública y entregas constantes permiten una evaluación continua del alumnado, aunque existen ítems de entrega y evaluación.

La docencia en estos TA se completa con una fase final individual 0 en grupos muy reducidos donde se trata problemas específicos de proyecto y su materialización, con un elevado grado de profesionalidad.

La constante contraposición entre propuestas creativas y limitaciones técnicas o normativas; la búsqueda de nuevos límites tecnológicos a las propuestas creativas; y la contraposición interdisciplinar enriquece el proceso de aprendizaje y conforma una metodología de resolución. Y, desde luego, gratifica y mejora la docencia y formación del profesorado. 


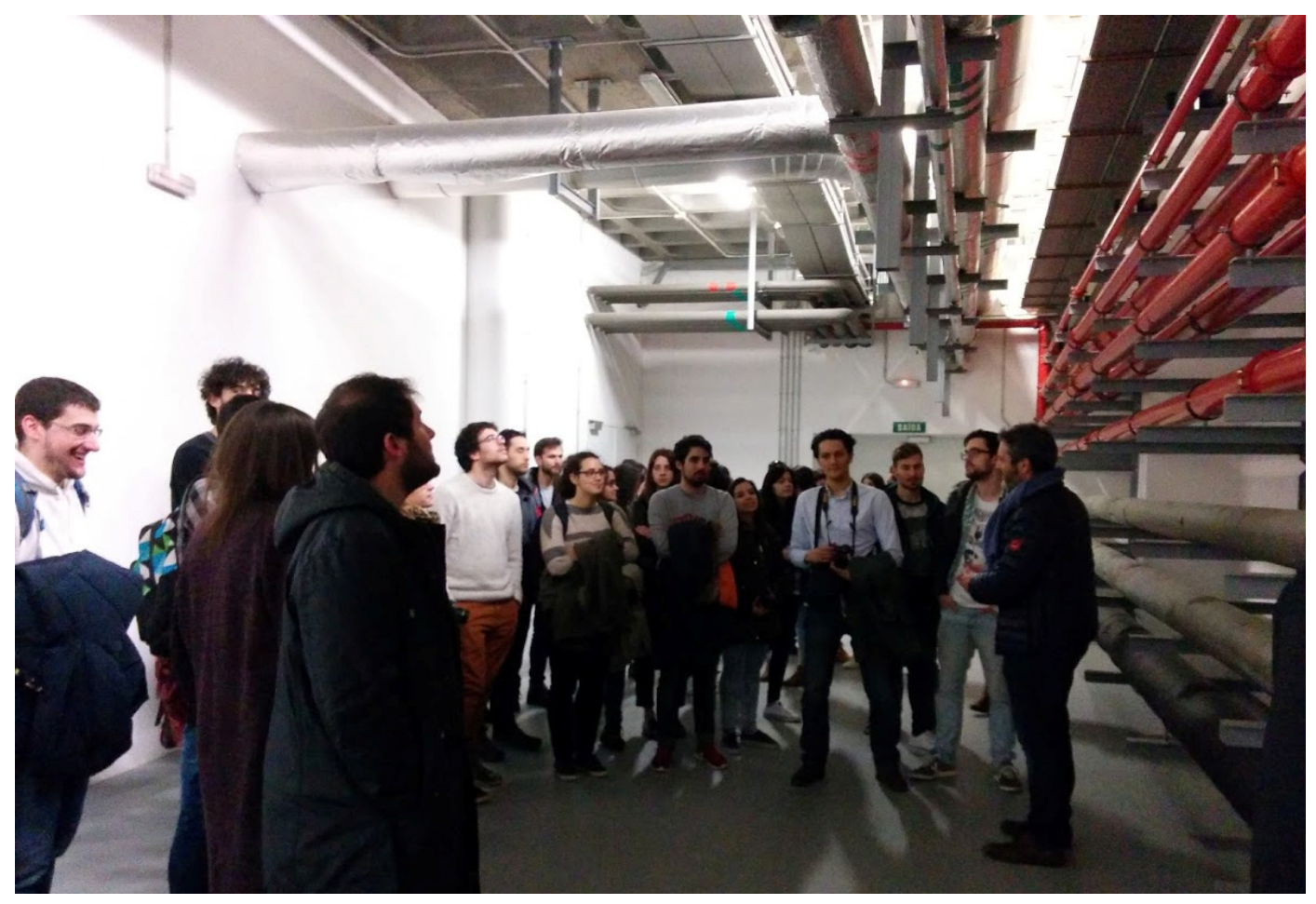

Imagen 3 Visita a las instalaciones de la Ciudad de la Cultura. Santiago de Compostela. Foto de la autora.

\subsection{Evaluación del TA}

La evaluación es continua, aunque la coordinación del TA establece unos criterios y fechas de presentación de los trabajos. Concretamente, en cuarto curso, la presentación era única en Moodle de la asignatura de proyectos, a donde acceden todos los profesores para evaluar en sus respectivas asignaturas el trabajo presentado por el estudiante. No existe una calificación del TA en grado.

Finalmente, en cuarto curso entre otros, se celebra una Junta de evaluación donde se contrastan criterios, calificaciones, desarrollo del semestre, disfunciones y propuestas para el siguiente semestre. Asimismo, se constata las condiciones del plan para cursar conjuntamente todas las asignaturas del TA. 


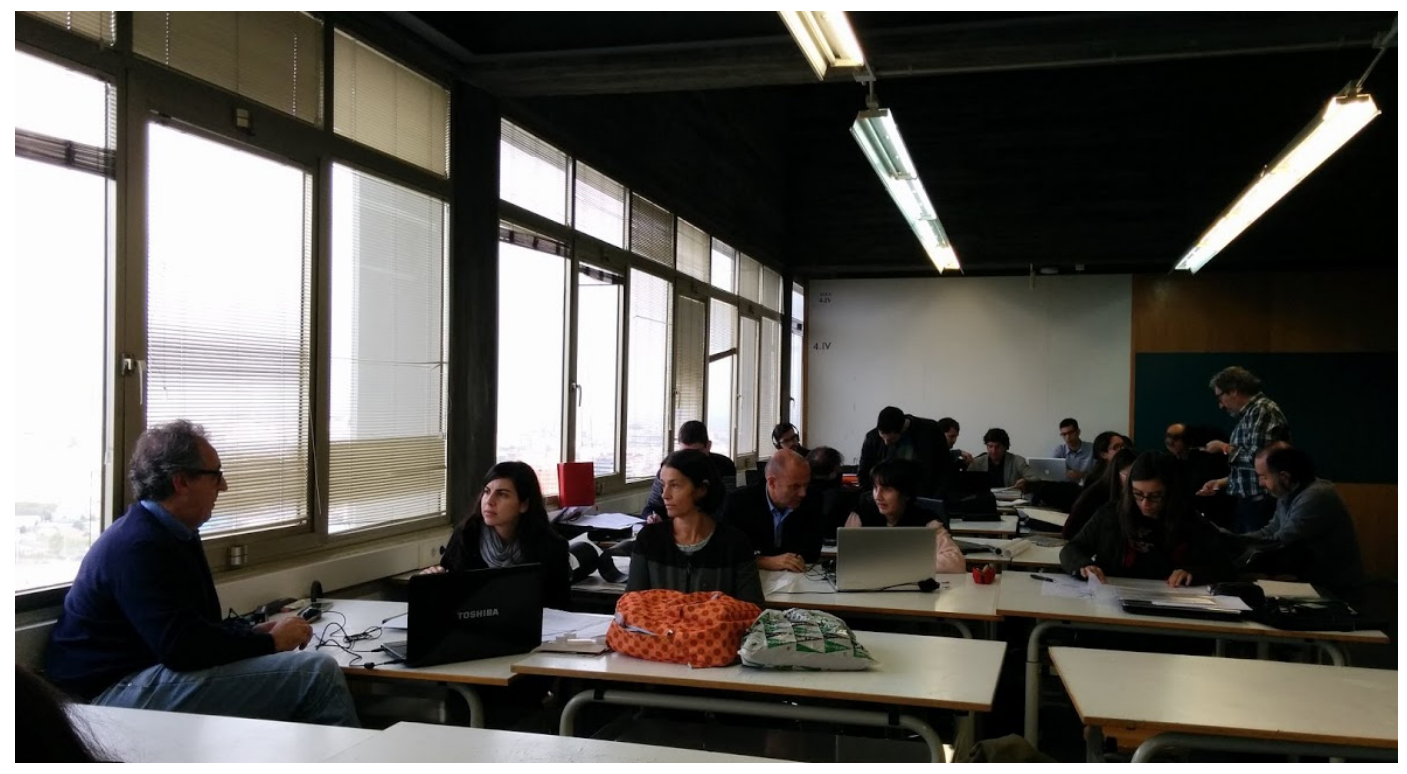

Imagen 3 Trabajo en el taller de arquitectura, aula gráfica ETSAC.

Foto de la autora.

En el caso del TA de PFC la evaluación del grupo de profesores se concreta en un apto o no para su defensa ante un Tribunal Universitario en el que deberá incluirse al menos un profesional de reconocido prestigio propuesto por las organizaciones profesionales (orden EDU2075/2010), que valorará de aprobado a matrícula de honor el trabajo, tras comprobación administrativa.

La fortaleza de una enseñanza transversal y unitaria con las enriquecedoras oportunidades del contraste opiniones, experiencias y experiencias de docentes, no oculta las debilidades del TA. Es evidente la existencia de diferentes disfunciones entre los cursos (como expone LópezBahut, E. vii) y entre los propios TA de PFC. Ya hemos citado la condición de matrícula para garantizar la horizontalidad y, desde luego, la falta de coordinación entre profesores 0 su incompatibilidad con otros docentes conforman las debilidades más significativas detectadas en los TA. 
La implantación de este modelo docente ha sido posible con el apoyo del Rectorado de la UDC, asumiendo propuestas y buscando soluciones como la posibilidad de simultanear varios docentes en una misma clase.

\section{RESULTADOS}

La estructura en diferentes disciplinas no se ha modificado sustancialmente en el Grado de 2010 y en el Grado + Máster de 2015, siendo la estructura de TA la innovación trascendental y diferenciadora introducida respecto al título de Arquitecto anterior.

La docencia en TA, no siendo la única causa, sí puede entenderse como fundamental en la mejora de las tasas de las titulaciones adaptadas a Bolonia. Nos solamente en la tasa de graduación, sino también en los resultados obtenidos curso a curso.

\begin{tabular}{|l|l|l|l|}
\hline Concepto & Titulación & $2015-16$ & $2016-17$ \\
\hline \multirow{2}{*}{ Aprobados/matriculados } & Grado Arquitectura & $21 / 222$ & $18 / 165$ \\
\cline { 2 - 4 } & Master Arquitectura & $34 / 34$ & $25 / 43$ \\
\hline \multirow{2}{*}{ Tasa de graduación } & Grado Arquitectura & $9,49 \%$ & $10,91 \%$ \\
\cline { 2 - 4 } & Master Arquitectura & $100 \%$ & $58,14 \%$ \\
\hline Tasa regularizada de & & $55 / 222$ & $43 / 165$ \\
graduación & $\mathbf{2 4 , 7 7 \%}$ & $\mathbf{2 6 , 0 6 \%}$ \\
\hline
\end{tabular}

Tabla 4 Datos obtenidos de Datawarehouse

A este resultado contrastable se suman otros más intangibles pero fundamentales: la colaboración entre profesores de diferentes especialidades se ha incrementado.

\section{CONCLUSIONES}

El funcionamiento de los Talleres de Arquitectura necesita de grupos de docentes afines, con perfiles profesionales capaces de escuchar y contraponer opiniones o criterios abiertamente, sin dogmas. El compromiso de los docentes, incluso mucho más allá de sus obligaciones docentes, es la base de este sistema de Taller de Arquitectura. 
Es una condición inexcusable la homogeneidad en las asignaturas cursadas por el estudiantado para el funcionamiento del TA.

La madurez obtenida por una larga duración de los estudios del Título de Arquitecto se sustituye por un aprendizaje dirigido por la transversalidad y unicidad de objetivos con una fuerte dedicación del profesorado y de los estudiantes, más allá de la estructurada por los ECTs.

Este sistema de docencia integrado se basa en la calidad y cualidad de las competencias adquiridas.

Seguramente los mejores resultados se obtienen en los últimos cursos y, concretamente, en el Taller de Arquitectura de PFC.

La docencia colaborativa en concursos competitivos de estudiantes como concurso internacional "Multiconfort house" ha permitido obtener resultados de calidad y excelencia para la ETSAC y para los estudiantes que la componen.

La naturaleza de asignatura de estos TA es un aspecto a estudiar. Sorprende que esta cualidad no sea un problema en PFC y si en el resto de semestres.

\section{REFERENCIAS}

- Lastra, E. A. (1971). Taller total en la facultad de arquitectura y urbanismo de la universidad nacional de Córdoba. Córdoba, Argentina: Universidad Nacional de Córdoba.

- López-Bahut, E. (2016). El "Taller" como herramienta docente en el ámbito del Proyecto Arquitectónico. García Escudero, D., Bardí Milà, B., Domingo Calabuig, D., (Eds.) IV Jornadas sobre Innovación Docente en Arquitectura (JIDA'16), Escuela Técnica Superior de Arquitectura de Valencia, 20 y 21 de Octubre de 2016".(pp. 218225). Valencia: UPV; Barcelona: UPC.

- Vitruvius Pollio, M., Barbaro, D. (1567). M. Vitrvvii Pollionis. De architectura libri decem. Venetiis [Venecia]: apud Franciscum Franciscium Senensem, \& Ioan. Crugher 
Germanum. Tratado original escrito por Marcus Vitruvius Pollio entre los años 27 a. C. y 23 a. C.

- ETSAV, Escuela Técnica Superior de Arquitectura del Vallés. Titulación de Arquitecto Plan 1993. Disponible en https://etsav.upc.edu/ca/estudis-old/titol-arquitecte [Consulta: 2 noviembre de 2017].

- ETSAC, Escuela Técnica Superior de Arquitectura (2010). Memoria General del Título. Graduado en Arquitectura por la Universidad de A Coruña. Disponible en http://etsa.udc.es/web/wp-content/uploads/2014/05/Bolonia-1-Plan-de-estudos-Graoen-Arquitectura.pdf [Consulta: 2 noviembre de 2017].

- ETSAC, Escuela Técnica Superior de Arquitectura (2015). Memoria General del Título. Graduado en Estudios de Arquitectura por la Universidad de A Coruña. Disponible en https://app.box.com/s/aj82wbmuakqfvv2mxn3kdzwc60oaeqld [Consulta: 2 noviembre de 2017].

- ETSAC, Escuela Técnica Superior de Arquitectura (2015). Memoria General del Título. Máster Universitario en Arquitectura por la Universidad de A Coruña. Disponible en https://app.box.com/s/8yw11gcyzwstkzvmgb08s2z9k9138v74 [Consulta: 2 noviembre de 2017].

- ETSAC, Escuela Técnica Superior de Arquitectura (2015). Reglamento del Proyecto Fin de Grado. Graduado en Arquitectura por la Universidad de A Coruña. Disponible en http://etsa.udc.es/web/wp-content/uploads/2015/06/20150601-Reglamento-PFGGraduado-en-Arquitectura.pdf [Consulta: 2 noviembre de 2017].

- ETSAC, Escuela Técnica Superior de Arquitectura (2015). Reglamento del Trabajo Fin de Máster: Proyecto Fin de Carrera. Máster Universitario en Arquitectura por la Universidad de A Coruña. Disponible en http://etsa.udc.es/web/wpcontent/uploads/2015/06/20150601-Reglamento-PFC-Master-Universitario-enArquitectura.pdf [Consulta: 2 noviembre de 2017]. 
' El actual modelo europeo tiene su fundamento disciplinar en el tratado "De architectura libris decem" de Vitrubio escrito a partir del año 27 a.C.

ii Tabla extraída del anexo 8.1 de la Memoria de verificación del Grado en estudios en Arquitectura 2014

iii Lastra, E. "Taller total en la facultad de arquitectura y urbanismo de la universidad nacional de Córdoba" FACULTAD DE ARQUITECTURA Y URBANISMO: Taller Total. Plan de Estudios, Universidad Nacional de Córdoba, 1971, reimpreso en 1975.

iv Mención 2011, Primer premio 2012, Primer premio 2013, Segundo premio 2015 y Primer premio 2016

${ }^{v}$ Memoria verificada Grado de Arquitecto por la UDC 2009. pp26

vi $\quad$ http://etsa.udc.es/web/wp-content/uploads/2015/06/20150601-Reglamento-PFC-MasterUniversitario-en-Arquitectura.pdf

vii Lopez-Bahut, E. 2016 